\title{
Intelligent Tutoring Systems by and for the Developing World: A Review of Trends and Approaches for Educational Technology in a Global Context
}

\author{
Benjamin D. Nye
}

Received: 31 December 2013 / Accepted: 31 August 2014 / Published online: 9 October 2014

(C) International Artificial Intelligence in Education Society 2014

\begin{abstract}
As information and communication technology access expands in the developing world, learning technologies have the opportunity to play a growing role to enhance and supplement strained educational systems. Intelligent tutoring systems (ITS) offer strong learning gains, but are a class of technology traditionally designed for most-developed countries. Recently, closer consideration has been made to ITS targeting the developing world and to culturally-adapted ITS. This paper presents findings from a systematic literature review that focused on barriers to ITS adoption in the developing world. While ITS were the primary focus of the review, the implications likely apply to a broader range of educational technology as well. The geographical and economic landscape of tutoring publications is mapped out, to determine where tutoring systems research occurs. Next, the paper discusses challenges and promising solutions for barriers to ITS within both formal and informal settings. These barriers include student basic computing skills, hardware sharing, mobile-dominant computing, data costs, electrical reliability, internet infrastructure, language, and culture. Differences and similarities between externally-developed and locally-developed tutoring system research for the developing world are then considered. Finally, this paper concludes with some potential future directions and opportunities for research on tutoring systems and other educational technologies on the global stage.
\end{abstract}

Keywords Intelligent tutoring systems $\cdot$ Digital divide $\cdot$ Systematic mapping study $\cdot$ Mobile learning $\cdot$ Barriers to adoption

B. D. Nye $(\bowtie)$

The University of Memphis, 365 Innovation Drive, Memphis, TN 38152, USA

e-mail: benjamin.nye@gmail.com 


\section{Introduction}

The Artificial Intelligence in Education (AIED) community is increasingly recognizing the importance of designing technologies with a global reach. One driving force may be that researchers are increasing their focus on the cultural facets of learning. However, the primary force may be increased information and communication technology (ICT) access in developing countries. This sustainable expansion of ICT worldwide is expanding the breadth of users who could benefit from educational software. Additionally, researchers outside of the traditional most developed countries have been building and adapting technologies targeting their regional and national needs. With these trends in mind, this paper looks at challenges and research directions related to intelligent tutoring systems (ITS) developed for and by the developing world. In looking at these trends, usage of ITS across a variety of contexts is considered, ranging from traditional formal settings (classroom settings) to emerging informal settings (home study on mobile devices). While this study focuses on ITS and adaptive systems papers, these findings tie into wider patterns that also have implications for a broad range of educational technology.

Dramatic shifts are projected for technology in developing regions over the next decade. Recent studies show that the digital divide is narrowing rapidly, driven by the expansion of broadband access in developing countries. Between 2005 to 2011, the percentage of households with Internet access in developing countries doubled from less than $10 \%$ to over $20 \%$ and is projected to reach $50 \%$ or more by 2015 (International Telecommunication Union 2012, p. 10). This level of growth would add nearly 1.75 billion Internet users, 500 million more than the combined population of all developed countries (Population Reference Bureau 2012). Because these areas struggle with shortages of qualified teachers and traditional educational resources such as textbooks, intelligent tutoring systems have the opportunity to play a pivotal role in supporting and supplementing their educational needs.

The ability of existing ITS architectures to address these challenges is unclear. Potential barriers for successful adoption of ITS in developing countries must be better understood, such as constraints due to data costs, mobiles as a primary information and communication technology (ICT), language support, and cultural values. To examine these issues, this research considered the current state of ITS research regarding its applications in the developing world. This study consists of four parts:

1. Identify barriers for ITS adoption in the developing world

2. Systematically review the level of ITS research focus placed on each barrier

3. Summarize current ITS research targeting each barrier

4. Compare and contrast ITS created by and for the developing world

The next section examines trends for technology access in developing countries and identifies barriers that significantly impact ITS suitability in these areas. These insights were used to design a systematic mapping study of the ITS literature examining the prevalence of recent research (2009-2012) that addresses barriers to ITS adoption. Only recent research was considered, to limit the review to potentially active projects. The results of this study provide insight into the challenges and solutions for ITS in the developing world. 


\section{Barriers to ITS in the Developing World}

Before considering the barriers to ITS in the developing world, it bears mentioning that the developing world is highly diverse, not a monolith. No universally agreed definition for a developing country exists, and development is probably better described as a multi-faceted continuum of wealth, opportunities, and social services. The most inclusive definitions of the "developing world" include any country not among the traditional most-developed countries (MDC), such as the World Bank's list of approximately 30 high-income Organization for Economic Co-operation and Development (OECD) countries or their larger list of 74 high-income countries (World Bank 2013b). However, there are obviously major divisions among the remaining countries. Countries often labeled as newly-industrialized, such as Brazil and Mexico, are in vastly different positions than least developed or unstable countries such as Sudan and Somalia. Additionally, large countries such as China, India, and even the United States have vast inequalities across regions and subcultures. This paper does not seek to reduce or minimize these differences, as every educational technology should ideally be tuned to the specific intended learners and educational ecosystem. Instead, this study has looked for general trends, problems, and solutions that have emerged in some developing countries and may be important to tutoring systems globally.

Barriers to educational technology are formidable, due to the large variety of stakeholders deeply invested in educational systems. This is further complicated in the developing world, where technology is often funded by and introduced from the most-developed countries. Figure 1 conceptualizes these stakeholders in terms of an ecological system for ITS. In terms of a Bronfenbrenner (1992) system, students have direct influences through their microsystems (family, peers, teachers, local administrators), indirect influences from the mesosystem (interactions between microsystem stakeholders), and macrosystem factors (infrastructure, governmental policies, cultures). Moreover, the developers of educational technologies such as ITS have their own ecosystem (e.g., collaborators, funding agencies, cultures). Cultural divides can exist between between technology sponsors, developers, host governments, and endusers. Availability and perceptions of appropriate educational technology must be communicated across these divides.

To identify barriers that primarily impact the developing world, the barriers noted in developing countries were contrasted against the barriers encountered in most developed countries. Barriers for most developed countries were drawn from Balanskat et al. (2006); Bingimlas (2009); Goktas et al. (2009); Lowther et al. (2008); and Riasati et al. (2012). These reviews focus primarily on formal settings in the US and Europe. Research in these contexts emphasized teacher and school factors, such as time constraints, in-service training, administrative support, match to teachers' pedagogical views, and teacher beliefs on ICT. Developing countries share these barriers, but have additional challenges as well.

Barriers in developing world contexts were drawn from Gulati (2008), who reviewed barriers specific to developing nations at that time, and Cassim and Eyono Obono (2011), who presented barriers relevant to the Kwa-Zulu Natal province of South Africa. Evaluations of ITS interventions in developing countries were also considered, including a multiple-user math tutoring in India (Brunskill et al. 2010), 


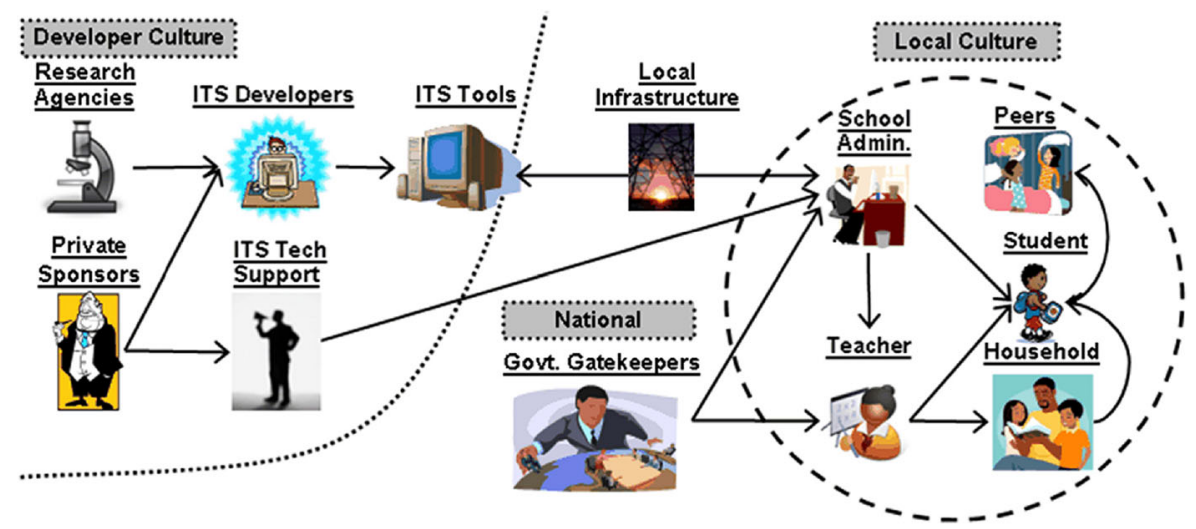

Fig. 1 Ecological System for Intelligent Tutoring Systems

literacy tutors in Ghana (Mills-Tettey et al. 2009), math tutoring in India (Banerjee et al. 2007), and Cognitive Tutor field studies in Latin America (Ogan et al. 2012). Based on this review, seven barriers to adoption were distinct to the developing world: 1. ICT hardware availability, 2. Electrical reliability, 3. Internet reliability, 4. Data costs, 5. Students' basic ICT skills, 6. Language, and 7. Lack of culturally appropriate content. Before ITS research can be developed for the majority of the world, these barriers must be understood.

Of these, lack of ICT hardware remains the primary barrier in the developing world. As mobile phones are the primary computing platform in these areas, lack of software targeting these devices is a related problem. Information and communications technology (ICT) has favored wireless mobile access over wired access (e.g., landlines, cable) in developing nations. Mobile platforms for ITS delivery are a growing topic in ITS research, but the constraints of a developing country on data or device features may present unforeseen problems. Sustainable educational technologies need to heavily leverage existing technologies, such as by targeting mobile platforms or through sharing devices.

Unreliable electrical and Internet infrastructure is a second technological barrier. Most-developed countries seldom struggle with their infrastructure, but this problem continues in developing areas, particularly in rural areas. With that said, despite common misconceptions, electrical access is seldom a primary barrier in developing countries overall. However, slow and inconsistent Internet infrastructure is a significant issue in many areas. Inconsistent Internet can be particularly vexing for a classroom setting; it is impossible to plan a lesson around an inconsistent resource. Even a relatively small rate of failure (e.g., $3 \%$ ) could ruin a week of classes each year. Slower data speeds also have implications. While over $90 \%$ of the world's population is covered by $2 \mathrm{G}$ mobile data signals (usually 2-3 times faster than a dialup modem), only $45 \%$ of the world is covered by mobile broadband (3G or better) and, as of 2011, there were only 8 mobile broadband subscriptions per 100 people in the developing world (International Telecommunication Union 2012). Updated figures indicate that this will be closer to $21 \%$ by the end of 2014 (International 
Telecommunication Union 2014). This has significant implications for transitioning data-intensive web applications. Data costs were not noted as a significant barrier for classroom use, but could significantly impact informal and home use.

Additionally, research has shown that experience and perspectives on ICT play a significant role in successful adoption. Basic ICT skills by learners were not a major factor in classroom settings but posed major hurdles for individual ICT use. Classroom-based systems, such as Cognitive Tutor (Casas et al. 2011), LISTEN (Mills-Tettey et al. 2009), and others (Gulati 2008), have found that lack of basic ICT skills was mitigated by having already set-up computers and peer support. However, Gitau, Marsden, and Donner (2010) found that mobile-only Internet users in South Africa faced serious hurdles setting up their devices for Internet access and required a combination of tutorials and user groups to reach web applications. This means that informal learning environments may be particularly sensitive to students' basic ICT skills.

Language barriers and culturally appropriate content were also considered significant issues. The dividing lines between these are not always completely clear. Figure 2 notes different levels of language and cultural localization from the standpoint of semiotics, which considers how signs are grounded in cultural meanings (Eco 1979). Language barriers primarily consider syntactics (relationships between signs) and semantics (meaning of isolated signs), which are used in translation. Syntax and semantics determine how knowledge is represented as educational content. From a language standpoint, educational technology unavailable in languages used locally for education will be a non-starter. Translation includes not only languages, but also other types of signs and symbols. Even "obvious" icons can be unfamiliar: in some cases, children have had trouble using software because hand-held microphones shown on icons were unfamiliar in their region. Without sufficient prior knowledge, some abstract concepts are hard to represent using icons, such as a "record" button for recording audio (McKnight and Read 2009).

Fig. 2 Levels of Language and Cultural Barriers

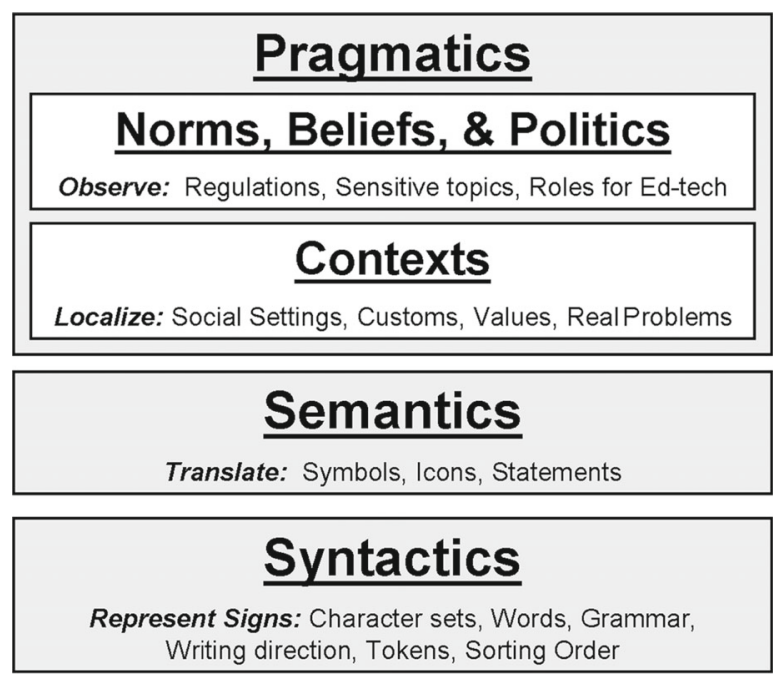


Beyond these fundamentals, cultural pragmatics emerge: the contexts, situations, and motivations that underlie the use of language and cultural symbols. Pragmatics constrain which contexts can be meaningfully presented in educational content. For example, culturally-grounded scenarios such as ice cream booths or hamburgers are entirely unfamiliar in some regions. These types of scenarios can prevent content from being culturally relevant. This introduces a conflict between cross-cultural technologies and anchored learning, where facts are grounded in realistic examples (Bransford et al. 2000). Anchored problems are harder to transfer across cultures, because "real life" examples assume certain prior life experiences.

Finally, educational content is constrained by the surrounding norms, beliefs, and political environment. These pragmatics impact why content should (or should not) be presented. Cultural sensitivities and taboos vary significantly: some topics may be relevant but too controversial for a local educational context. For example, a math problem describing a woman driving a car would be a live-wire issue in some areas. Educational content may even be explicitly regulated, such as when laws mandate maps with particular interpretations of disputed borders. For domains such as history, differences in perspective could pose major barriers to sharing content at all. Views about the role of educational technology may also impact the types of uses that are acceptable (e.g., in-class practice, team projects, after school, web homework, etc.). Standardized assessments fall under this category as well. Content that corresponds with key tests and curriculum standards has an advantage over generic materials. These top-down pressures from the larger cultural macrosystem impact the ecological fitness of educational technology.

Overall, there are significant additional barriers to designing or transitioning educational technology for developing countries. Intelligent tutoring systems, one of the most effective educational technologies, may be particularly affected by these issues. Compared to content-agnostic technologies such as learning management systems, ITS include large amounts of "baked-in" materials (e.g., hints, curriculum units). These materials offer a major advantage for teaching in resource-limited environments: they provide individual support to students, where large class sizes and shortages of trained teachers might make this otherwise impossible. However, traditional ITS research has focused primarily on WEIRD (Western, Educated, Industrialized, Rich, Democratic) countries. As such, it is important to identify how much focus has been placed on each issue and the proposed solutions to these barriers.

\section{Systematic Mapping Study: Recent ITS Literature Addressing Barriers}

A systematic study of recent ITS publications was conducted to identify the prevalence of literature that notes problems or solutions related to each barrier. Systematic mapping studies are similar to systematic reviews, except they employ broader inclusion criteria and are intended to map out topics rather than synthesize study results. The study presented here covers recent ITS work: articles and conference papers published no earlier than January 1, 2009 and indexed before January 1, 2013. A four year span was chosen to limit the study scope to potentially active projects and to balance 
the number of Intelligent Tutoring Systems (ITS) and Artificial Intelligence in Education (AIED) conferences, as these are key publishing venues for the field. This time frame was chosen to limit the review to potentially active projects, since projects with no publications in the last 5 years are likely inactive. This review followed guidelines for systematic mapping studies contained in Petersen et al. (2008).

The primary aim of the current study was to examine how much of the recent ITS literature addresses each barrier in the developing world. This study posed the research question: "What fraction of ITS research addresses each of these barriers to adoption?" A large scale review of ITS work related to the developing world has never been conducted, so these topics require significant background to explain their significance and potential solutions.

\section{Inclusion Criteria}

Inclusion criteria were based on the following question: "Does the paper describe original research on ITS design, enhancements to an existing ITS design, studies using an existing ITS, or analysis of data collected in a study using an ITS?" To identify an ITS, this study used the inner-loop criterion, which requires stepbased tutoring (i.e., adaptive support while working on a task). This is based on VanLehn (2006), who identified two types of adaptivity found in common across intelligent tutoring systems: an outer loop and an inner loop. The outer loop determines the next learning task that a learner performs, such as a math problem or a passage to read. Typically, the outer loop is used to keep tasks within the learner's zone of proximal development (Vygotsky 1980). Since the outer loop does not need to provide just-in-time feedback, domain-agnostic machine learning techniques are often utilized for this adaptivity. On the converse, the inner loop helps the learner while they are working on a given learning task. Inner-loop adaptivity provides step-specific scaffolding, such as sequences of hints, error-correction feedback, and cues that orient the learner to important parts of the current problem state. The inner loop is a defining characteristic of intelligent tutoring systems: while a variety of other systems employ outer loops (e.g., adaptive e-learning systems), adaptive support on an ongoing task is distinct to ITS. This fine-grained support is significant: a meta-analysis found that systems with step-based adaptivity (inner loops) produced higher learning gains than those with only an outer loop (VanLehn 2011).

Papers that met the primary inclusion criteria required an inner loop, where the system to provided hints and/or feedback intelligently. Since this criterion is not always straightforward to apply, a second inclusion category of "adaptive learning systems" was employed. This category collected papers at the fuzzy fringe of tutoring systems that use only an outer loop (e.g., adaptive hypermedia) or only provided rudimentary feedback (e.g., game-based learning with simple feedback). This determination is based entirely on the functionality presented, not merit (i.e., ITS are not necessarily "better" than adaptive learning systems). It should be noted that, while the study should be relatively comprehensive for ITS, the search process was not designed to comprehensively cover adaptive learning systems, which were analyzed only to consider the fringe of inclusion criteria. 
Units of Analysis: Papers and Architecture Families

The study considered two complementary units of analysis: papers and ITS architecture families. These provide different indicators of the amount of research conducted. Though examining individual papers assesses the total volume of research published on each topic, this biases the sample toward large, established architectures that publish extensively. To address this, another approach classified each paper by the family of ITS architectures (e.g., Cognitive Tutor, AutoTutor, etc.), with each family being treated as a single unit of analysis, to better capture how many lines of architectures address each barrier.

Avoiding bias in categorization of papers into architectures was a thorny issue, as architectures often evolve or are renamed over time. If any paper explicitly stated that its design extended an older architecture, all articles referring to both architectures were considered part of the same family. Additionally, ITS produced by the same research group and sharing similar features were also grouped together. Unnamed architectures were assumed to be unique and were classified by their author names and year, unless there was a clear overlap in both authors and ITS architecture design. In practice, this was not a significant issue, as most papers either used a well-established architecture or described a one-off project described by only one paper.

\section{Search and Screening Methodology}

This review searched major citation aggregators that cover key journals and conferences that publish ITS research. Results were aggregated from Thomson-Reuters Web of Science, ACM Digital Library, IEEE Xplore, and ERIC. Searches for publications were based on the phrase: "intelligent tutoring system" OR "intelligent tutoring systems". The broadest possible search settings were used: all available holdings in each index were searched, using full-text search rather than keywords if available. This generated a citation set of 2647 journal and conference publications. Short papers and demonstrations were included in this review, as these papers occasionally address aspects of an ITS that are otherwise unpublished. The full text of 2586 papers was evaluated. 19 papers were excluded because their text was not written in English. An additional 42 papers could not be located in available institutional holdings or by inter-library loan. As 33 of these were clearly irrelevant based on their abstracts, only 9 potentially relevant papers could not be reviewed in full.

Possible sources of search bias include the keywords used to select publications for review. Using "intelligent tutoring system(s)" as a search term could exclude adaptive or personalized learning systems that might qualify as tutoring systems but were not referred to as such. Thus, the review is biased toward communities of practice that use this terminology, though it would seem unlikely that any large community of practice would perform similar research without any references to ITS. A follow up search indicated that "tutoring system(s)" might have yielded approximately $10 \%$ more citations, so this may be a more comprehensive term to use for future studies, though these additional citations did not appear to be characteristically different from those evaluated. Secondly, only papers in English were reviewed. However, research 
from over 60 countries was represented, likely allowing a reasonable representation of the state of the field internationally. Finally, the choice of citation indexes may have impacted the findings, as unindexed journals, books, forthcoming work, or grey literature may show slightly different patterns.

\section{Classification Criteria}

Each paper meeting inclusion criteria was evaluated based on eight Boolean classification criteria based on each barrier:

1. Student Basic ICT Skills: Does the ITS research address usability by learners without basic computer experience or skills?

2. Hardware (Sharing): Does the ITS research address lack of hardware or multiple users sharing a single computing device?

3. Hardware (Mobile): Does the ITS address mobile devices, such as a mobile application or mobile version of a website?

4. Data Costs: Does the ITS research address reduced or optimized data transmission over a telecommunications carrier?

5. Electricity: Does this paper describe electricity problems or methods to avoid such problems?

6. Internet: Does the ITS research address unreliable Internet connectivity?

7. Language: Does the ITS design address multiple language support or describe features to facilitate language localization?

8. Culture: Does the ITS design include cultural features, cultural content, or features to facilitate cultural localization?

As these are not focal topics of the ITS community, criteria were applied broadly. Papers that addressed these topics in any fashion were included, even if they briefly noted the barrier as an obstacle (e.g. "due to insufficient computers, students had to share"). This determination was based upon the full text of the paper. However, raw publication counts are biased toward groups who publish more extensively. For that reason, papers were grouped into families of architectures as a secondary analysis. For this measure, an architecture needed only one paper stating the existence of a feature to be classified as having that feature. A paper was categorized as part of a family of architectures if it used a stated ITS, used an ITS developed by the same research group that extended or succeeded a given architecture (e.g. a pedagogical agent added to an existing architecture), or analyzed data produced by that architecture. This gives an estimate of how many different family trees of ITS research have examined each topic recently. If any paper based on an architecture met the criteria, the architecture was classified as meeting the criteria (i.e., a Boolean union).

Additionally, the country or countries primarily affiliated with each paper and architecture were collected. For each paper, a list of countries was collected that consisted of the country for the first author's institutional affiliation plus the set of countries which were noted as evaluation sites. For the vast majority of papers, this produced only a single country affiliation (i.e., researchers evaluated their system within their institution's country). However, papers that specifically focused on transitioning ITS between countries had up to four countries affiliated (e.g., project origin 
country plus three evaluation sites). As with the other criteria, the list of affiliated countries for an ITS architecture was calculated as the union of the country sets from the individual papers on that system.

\section{Mapping Study Results: The Geography of ITS}

A total of 815 papers on ITS fit the primary inclusion criteria, as well as an additional 240 papers on adaptive learning systems (ALS). This analysis, despite covering a greater breadth than Blanchard (2012), also shows a strong WEIRD (Western, Educated, Industrialized, Rich, Democratic) bias. Approximately $75 \%$ of papers had a first author in such a country and, if data was used, it was collected only in WEIRD countries. Figure 3 shows a heat-map of the log of publication counts for each country across all 1055 papers included. Papers with multiple country affiliations were counted once for each. Darker shades indicate higher publication counts, with the lightest countries having only a single publication during the period, and the darkest with hundreds of papers.

The US was disproportionately represented, even adjusting for population, with 393 papers. The next-highest countries (China, Spain, United Kingdom, Canada, and Taiwan) each had between 67 and 50 papers. The next tier (10-30 papers) consisted primarily of Western countries (Germany, Greece, France, Australia, New Zealand, etc.) and large developing economies (Mexico, India, Brazil, Malaysia). While most countries' papers described ITS (United States, $95 \%$; Canada, $92 \%$; Germany, 80 $\%$; UK, $82 \%$ ), other countries had fewer ITS papers (Italy, $32 \%$; Spain, $42 \%$; Taiwan, $54 \%$ ) and more adaptive systems at the fringe of this analysis. In total, 52 countries conducted research with at least one ITS paper in this analysis, with an

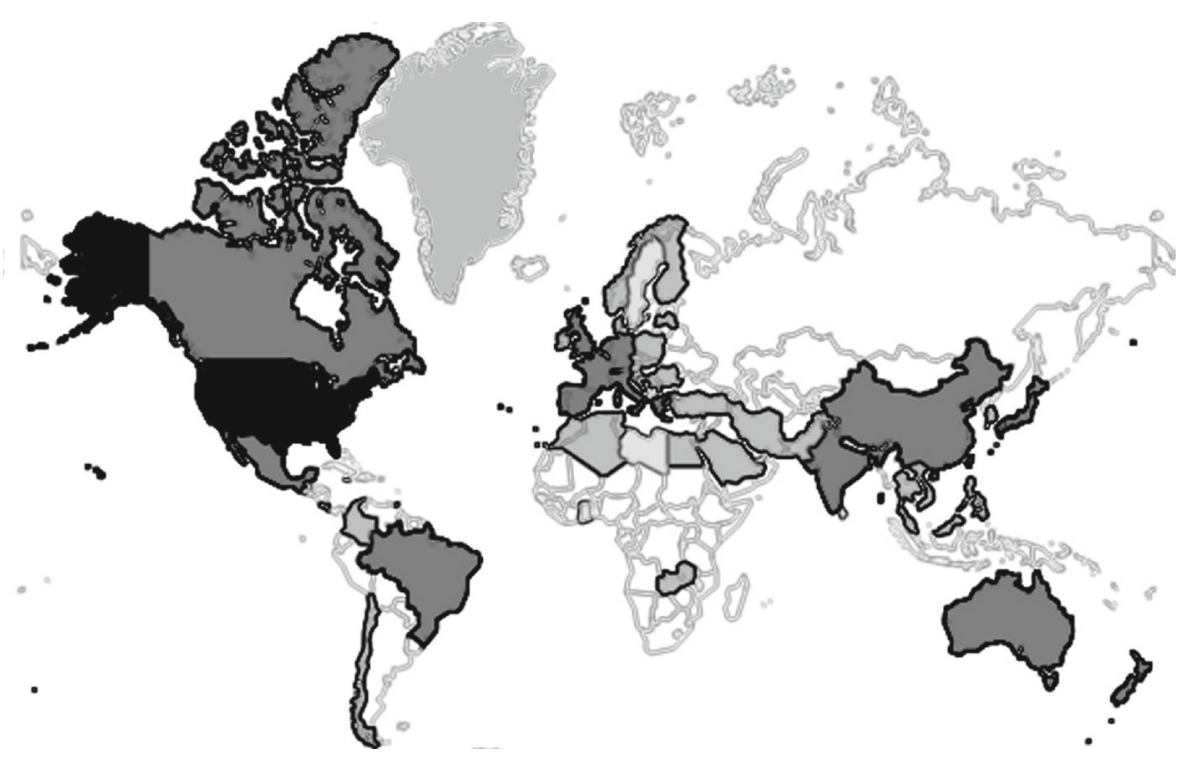

Fig. 3 Country Affiliations for ITS and Adaptive Systems Reviewed 
additional 9 countries contributing at least one paper on an adaptive system. Surprisingly, no papers from Russia met the criteria. The reasons for this are unclear, but publication in Russian-language journals or different research priorities might explain this finding. A full breakdown of the paper counts by country is contained in the Appendix.

Unsurprisingly, smaller countries (by population) tended to produce fewer papers. However, the nominal GDP (gross national product) was a better indicator of output. World Bank GDP figures were used (World Bank 2013b). A high correlation $(\mathrm{r}=0.88, \mathrm{p}<0.001)$ was found when considering the relationship between 2012 country GDP and the number of relevant papers over the period, as shown in Figure 4. GDP (the x-axis) is shown on a logarithmic scale to accommodate the wide disparities in income. To note, while the curve in Fig. 4 appears exponential, it is actually linear (the curvature is due to the logarithmic scaling of the x-axis). A total of 186 countries with recent data (2008 or later) were included and their most recent data was used (almost entirely 2012 data). A few representative or notable countries are tagged on the graph, for reference. With the exceptions of Russia and Indonesia, every country with at a GDP over 500 billion dollars had at least one paper that met inclusion criteria as an ITS or adaptive learning system. The relationship of publication counts as a function of GDP was highly linear (20 papers per trillion dollars GDP, after the first 66 billion dollars of GDP), and implies that research funding may be a limiting factor.

Figure 5 offers a slightly different view on this effect, plotting the number of included publications per million people as a function of the per-capita nominal GDP.

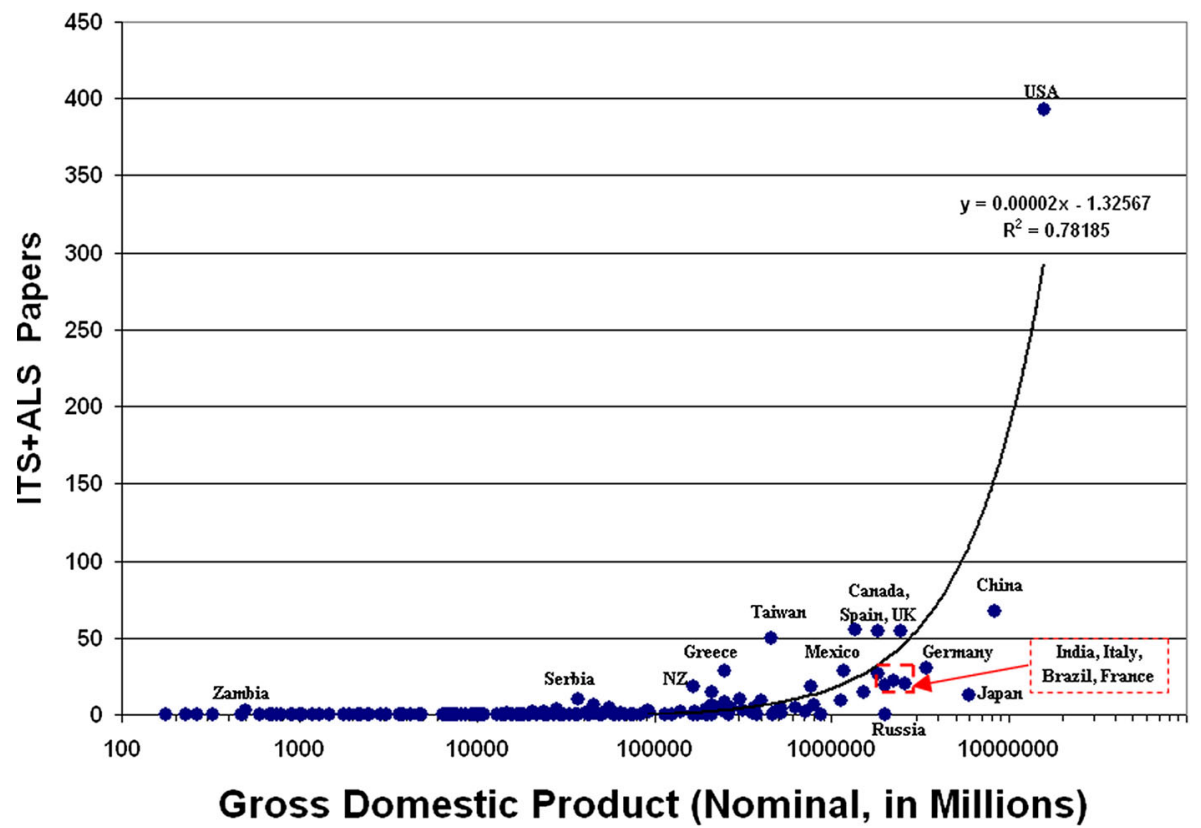

Fig. 4 Number of Papers Reviewed, By Country GDP 


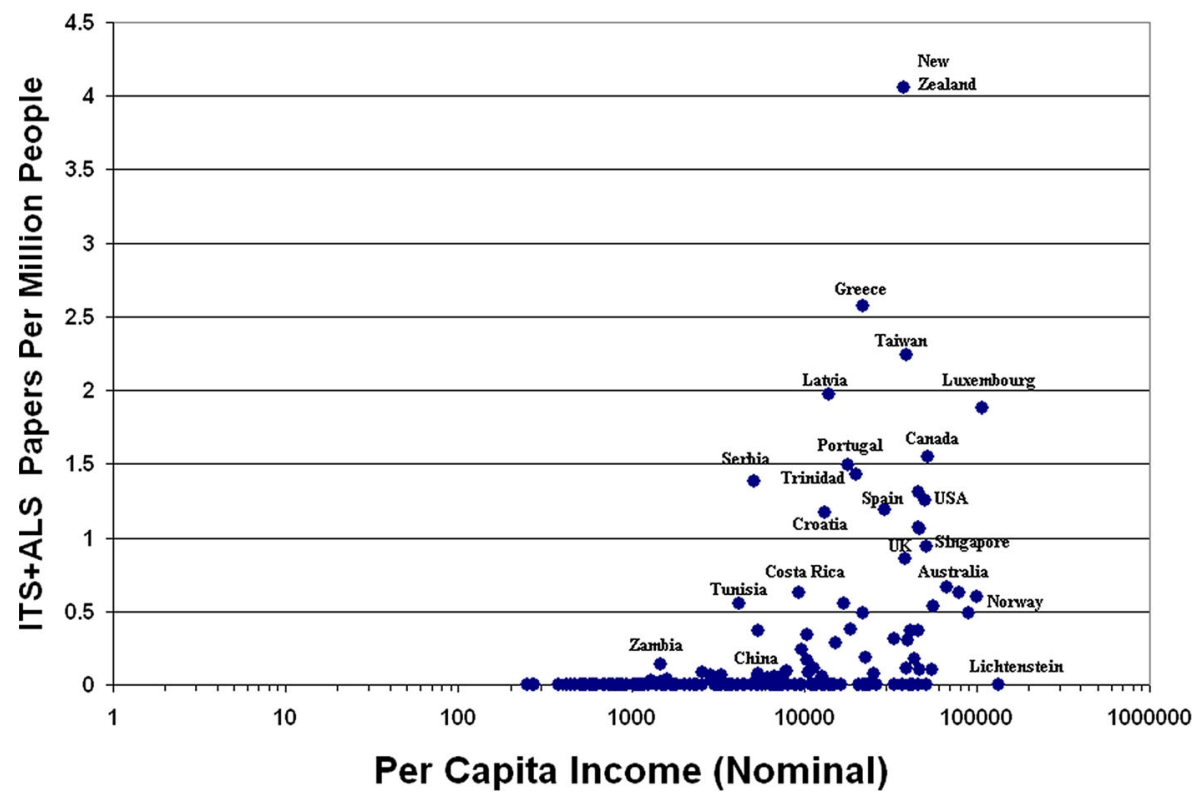

Fig. 5 Number of Papers per Million Capita, by Country per Capita GDP

While this approach has a high variance for small countries, it shows the impact of per-capita wealth on ITS research published internationally (and possibly research more generally). The United States is less of an outlier in this analysis, showing that its high output is significantly related to its larger size. Below a per-capita income of about $\$ 3,500$ USD, the included papers per capita bottoms out and the rate of publications is very low per capita. Zambia was a notable outlier with two ITS publications identified, despite a per-capita income close to \$1,400. The dropoff in Fig. 5 appears to be due to two effects. First, most smaller developing countries produced no papers or only one paper. Second, while larger emerging economies produced more papers, their output was still small relative to their population size. This indicates a generally low prevalence of ITS research focusing on developing nations.

While ITS research appears to be dominated by Western countries (and the United States, particularly), the map in Fig. 3 clearly demonstrates that a significant amount of ITS research is being conducted outside of the traditional WEIRD countries in absolute terms. In total, about $19 \%$ of reviewed papers (and $16 \%$ of ITS papers) included countries outside of the World Bank's list of high-income nations plus Taiwan, which is not officially categorized (World Bank 2013a). Most of this research is occurring in large emerging economies such as China, Mexico, India, Brazil, Malaysia, and the Philippines. Additionally, non-Western economically-advanced countries in Asia (e.g., Japan, South Korea, Taiwan) and countries at the fringe of the euro-zone (e.g., Serbia, Turkey, Croatia) are also producing significant volumes of research on the topic. 


\section{Mapping Study Results: Barriers to ITS}

Table 1 shows the results of the mapping study on developing world barriers. The first row shows the raw results, which are the percentage of ITS publications that address each barrier. The second row displays the results for unique ITS architecture families, such as Cognitive Tutor (Koedinger and Corbett 2006) and AutoTutor (Graesser et al. 2005). The third row displays the results for "Major" ITS architectures, those with more than 10 papers published during the study period. These architectures are highly influential and account for 290 of 815 papers on tutoring systems. The final row contains the results for the 240 adaptive learning system papers reviewed as part of this analysis. The numbers for families of adaptive learning systems are not presented because families were small (5 or less papers) and had nearly identical findings as those for the individual ALS papers.

Overall, a very small number of recent ITS papers approached any of these topics ( $<10 \%$ for most categories and samples, excepting papers from major ITS families discussing mobile access, Internet reliability, culture, and language). Even fewer papers addressed these topics in any depth. For comparison, over $45 \%$ of papers in the sample addressed student motivation (e.g., affect, games, etc.), and over 14 $\%$ considered student affect alone. Of these categories, mobile devices, culture, and language support received the greatest focus and were noted in over $4 \%$ of papers. The higher numbers for language may be slightly misleading, however, as many of these systems addressed second-language learning (i.e., language was the domain). While mobile support was the most prevalent category, the focus was still low compared to their prevalence. While more people in the world use a smartphone than a computer (desktop or laptop), almost $95 \%$ of ITS research still targets traditional computers. This is particularly important for emerging and developing economies, which currently appear to be leapfrogging traditional computers, similar to how they leapfrogged landline phones. Other topics were given even less attention. Students' basic ICT skills were considered by only $2 \%$ of papers. Hardware sharing, data transmission, electricity issues, and Internet reliability were barely addressed within the sample.

Examining the ITS families, similar patterns held. The ITS families shared the same ordinal relationships as the individual paper results, but with slightly higher percentages. Mobile platforms and students' basic ICT skills gained the most from this aggregation, which indicates that a larger number of systems are independently

Table 1 Percentages of ITS (and ALS) Addressing Developing World Barriers

\begin{tabular}{|c|c|c|c|c|c|c|c|c|c|}
\hline & $\mathrm{N}$ & $\begin{array}{l}\text { Student } \\
\text { ICT }\end{array}$ & $\begin{array}{l}\text { Hardware } \\
\text { Sharing }\end{array}$ & Mobile & $\begin{array}{l}\text { Data } \\
\text { Costs }\end{array}$ & $\begin{array}{l}\text { Electric } \\
\text { Infra. }\end{array}$ & $\begin{array}{l}\text { Internet } \\
\text { Infra. }\end{array}$ & Culture & $\begin{array}{l}\text { Multiple } \\
\text { Languages }\end{array}$ \\
\hline All ITS Papers & 815 & $2.21 \%$ & $0.98 \%$ & $5.77 \%$ & $0.49 \%$ & $0.25 \%$ & $0.73 \%$ & $4.90 \%$ & $3.93 \%$ \\
\hline ITS Families & 373 & $4.02 \%$ & $1.34 \%$ & $8.58 \%$ & $1.07 \%$ & $0.54 \%$ & $1.34 \%$ & $5.90 \%$ & $5.36 \%$ \\
\hline Major ITS & 12 & $16.7 \%$ & $8.33 \%$ & $33.3 \%$ & $8.33 \%$ & $0.00 \%$ & $25.0 \%$ & $41.7 \%$ & $16.7 \%$ \\
\hline All ALS Papers & 240 & $2.50 \%$ & $0.00 \%$ & $9.17 \%$ & $3.75 \%$ & $0.42 \%$ & $2.08 \%$ & $2.50 \%$ & $2.08 \%$ \\
\hline
\end{tabular}


addressing these topics. Major ITS families addressed more of these topics, on average. While it might be tempting to explain this by simply having more papers, and hence more chances to address a topic briefly, a pair of major ITS families considered them in depth. For example, a single article on transitioning the Cognitive Tutor to Latin America considered five: student ICT skills, hardware sharing, Internet reliability, language, and culture (Ogan et al. 2012). Secondly, the Tactical Language and Culture Training System (TLCTS) and related projects trained cross-cultural and language competencies, with support for mobile learning as well (Johnson 2010). These two systems account for half of the weight across the "Major ITS" row. The only topic addressed by multiple other systems was cultural factors, due to studies considering different subcultures within a country.

Based on these results, ITS research appears to have given these barriers little attention and would probably struggle in the developing world as a result. The following subsections briefly summarize the current literature on how ITS and other educational technologies are approaching these barriers.

\section{Student Basic ICT Skills}

Research on basic computing skills for students indicates significant differences between individual use and classroom use. Pilots of Cognitive Tutor and LISTEN Reading Tutor in the developing world found that students were able to navigate the software fairly quickly (Casas et al. 2011; Mills-Tettey et al. 2009). However, a study on mobile access in South Africa showed a much higher barrier to basic web use (Gitau et al. 2010). Two factors appear to mitigate lack of basic ICT skills by students: peer support and initial device setup (e.g., operating system setup, Internet and network connectivity). Peer groups can be very beneficial because even a small number of technically-adept learners can help inexperienced peers perform basic setup tasks. In some cases, technically-proficient learners have also helped teachers with limited ICT skills (Ogan et al. 2012).

In many ways, this is a support issue: users can learn how to use an ITS, but setting up a device is difficult. In classroom and institutional settings, this responsibility typically falls on technical support staff. Even where such support is limited, peer support sometimes mitigates this challenge. Informal and home contexts are more complicated, because technical support may be entirely unavailable. One solution is to simplify the system: Savvopoulos and Virvou (2010) approached elderly populations with low ICT skills by providing tutoring over interactive TV. The holein-the-wall experiments also sidestepped this hurdle by embedding Internet-ready computers in public spaces (Mitra and Dangwal 2010). However, mobile devices are the prevalent independent platform. On mobile platforms, community support such as libraries and schools may be pivotal to help install and setup ITS for home use.

\section{Hardware Sharing}

Sharing devices is a key technique for reducing barriers due to lack of hardware. From an ITS perspective, sharing a computer is a disruptive paradigm: most 
tutoring systems assume a 1:1 mapping of users to computers. Recent findings from the Cognitive Tutor project show that computer sharing accounts for over $60 \%$ of use in developing areas, with students leaving their own machines to share a machine instead (Ogan et al. 2012). LISTEN and other groups have had similar experiences: computer sharing, even when enough hardware is available, is characteristic of developing world ICT usage (Banerjee et al. 2007; Mills-Tettey et al. 2009). This has serious implications for the user model, which assumes that each machine is measuring the work of one person. Ogan et al. (2012) suggests modeling the classroom as a network of connected user models rather than individual models. Unfortunately, software techniques for disentangling multiple users sharing an input are not mature. Moreover, a software solution would reduce the power of knowledge assessments by adding uncertainty about user identity. User models that account for collaboration are worth exploring, but they may only offer a partial solution.

Existing ITS that share hardware have focused on using multiple inputs instead. MultiLearn+ split a laptop display into quadrants, each with their own keypad (Brunskill et al. 2010). Single Display Groupware went further, with a whole class sharing a single projection and one mouse per student (Alcoholado et al. 2012). The latter paradigm was problematic due to the complexity of managing dozens of mouse cords, but might be effective using wireless mice, clickers, or other input devices. Notably, neither of these field studies indicated that students exchanged or shared input devices extensively under these conditions. Using a single machine also facilitates modeling collaboration, since the data for multiple users is already in a single system. As such, embracing computer sharing might also mitigate some of the user modeling issues.

Hardware sharing offers strong advantages for classroom and institutional use (e.g., university computer labs). First, as noted, many cultures and students prefer to work in groups. Second, multi-user software can significantly reduce hardware costs. Since inputs are inexpensive compared to processors or displays, a four-user system like MultiLearn+ lowers the cost-per-student by almost three-quarters. Systems like Single Display Groupware that use cheap wireless clicker inputs might also be feasible, possibly reducing costs by an order of magnitude by adding a multiplier-effect to inexpensive hardware initiatives such as OLPC (Patra et al. 2007). Finally, fewer devices means less setup and maintenance, which can be a significant factor when technical support is limited.

Additionally, hardware sharing extends beyond multiple inputs to sharing across time and location. Turn-taking, such as in game environments, is a second established model for sharing a single machine across multiple users. At the school level, scheduling of computer labs (rather than dedicated computers in each classroom) is the most prevalent form of time-sharing practiced. Hardware sharing can also occur across locations and institutions. The "Learning Van" program drives a van-based "computer lab" with laptops and satellite Internet to different rural schools each day (Zualkernan and Karim 2013). Particularly for smaller schools that could not individually maintain computers and Internet access, this model for sharing is attractive. Designs to maximize hardware sharing across users, time, and location can leverage limited hardware to serve more students. 


\section{Mobile ITS}

Despite the expansion of mobile technology in the developing world, mobile ITS research was most prevalent in Western Europe (Virvou et al. 2012) and East Asia (Chu et al. 2010). A prevalent model for mobile ITS relies on dedicated applications (apps) designed for mobile devices. In the US, the Tactical Language and Culture Training System (TLCTS) for language learning supports a mobile application which retains the core elements of its original immersive ITS environment, including voice input and face-to-face conversation with virtual agents (Johnson 2010). Most of the reviewed mobile ITS research was designed for PDA's and higher-end smartphones, making it unlikely to transfer easily to low-end mobiles limited to $2 \mathrm{G}$ data speeds.

Transitioning a traditional ITS from desktops to mobile devices also has general hurdles due to the much smaller display and different input devices. While desktop applications use mice and keyboards, low-end phones offer a much more limited set of buttons. Voice input was also a common feature for mobile ITS focusing on language learning, allowing users to practice speaking new words out loud. This may imply that mobile ITS may benefit from substituting speech input in place of text input. Kumar et al. (2012) demonstrated that a speech-driven adaptive learning game was effective in India, but handling the regional accents required a corpus of local speech. In the same paper, they proposed an ambitious plan to use speech recognition for mobile sharing that could have significant implications. With that said, multiple users talking into a single mobile device may offer serious technological challenges. Speech recognition for a single user is already imperfect, particularly in noisy environments. Handling multiple speakers may prove impractical, especially if speaker identification is needed.

A second variant of mobile ITS are ubiquitous e-learning systems for universities, such as EDUCA in Mexico (Cabada et al. 2011). These systems provide strong outer loops using adaptive curricula and inner-loop functionality for subsets of the system. These mobile web gateways are a strong cross-platform delivery method, but they rely significantly on data transmission. EDUCA is notable because it installs an Intelligent Delivery Engine mobile application that locally executes tutoring modules downloaded from the server side. The server side can provide outer loop functionality using recommender system approaches (e.g., select resources that are likely to be useful). On the mobile device, a lightweight, unsupervised neural network helps to adapt the presentation of learning objects and quizzes for the learner. While this approach requires a dedicated mobile application, rather than just a web-browser portal, it offers the ability to download tutoring modules rather than relying on a persistent connection to the Internet.

Finally, a few mobile learning environments incorporate local data transmission using Bluetooth protocols. While no systems with full ITS capabilities used this approach, it has been incorporated into adaptive learning systems (Munoz-Organero et al. 2012; Puntambekar et al. 2009). Local data transmission may offer a powerful paradigm for future systems. Bluetooth and WiFi transmitters are becoming common even in lower-end phones, opening up the possibility of mobile-to-mobile sharing and networking between ITS instances. Much as early computer games used local area networks (LAN) to support multiplayer games, local data transmission could 
support computer supported collaborative learning and multiplayer game-based ITS. Most importantly, if combined with a system like EDUCA, learners could directly share tutoring modules (e.g., "This one I'm on right now has helped me a lot with that chapter. Here, let me share it with you."). While this topic has not been explored in depth, it warrants further investigation.

\section{Data Costs}

Data costs primarily impact mobile learning. Literature shows three main solutions: don't rely on data, use data in batches, and use data locally. Cognitive Tutor, EDUCA, and Learning Pills embody these concepts, respectively (Cabada et al. 2011; MunozOrganero et al. 2012; Ogan et al. 2012). Cognitive Tutor avoided these barriers because it can be installed and run as a standalone application on a PC (Ogan et al. 2012). One-time installs offer the appeal of a single transfer, such as through a CD installer, Internet download, or USB stick. This approach also works for mobile devices, such as by downloading a mobile application over WiFi or over a USB cable. This one-shot model is most useful for a synchronized institutional setting, such as a computer lab, where the ITS can be loaded onto all devices at once.

EDUCA allows users to receive ITS units as modules, allowing users to download them using cheap or free $\mathrm{WiFi}$ access rather than communicating wirelessly at runtime (Cabada et al. 2011). This approach allows a "well model" for data transmission. In many areas, running water is not available to every house, but people can go to a common well to obtain buckets of water. An analogous approach can be used for mobile devices, ranging from phones to laptops. Fixed-price broadband connections (e.g., cable, satellite dishes) can provide WiFi Internet and act as "wells" for mobile data. Systems like EDUCA can support downloading a tutoring module for later use, allowing learners to perform downloads when data is free or cheap.

Finally, Learning Pills relies on Bluetooth OBEX (OBject EXchange) protocols to allow an instructor's machine to directly transmit data to students' phones in the classroom (Munoz-Organero et al. 2012). While the Learning Pills sent fairly small study materials and messages to learners, the general concept could extend to more significant data packages (e.g., multiple megabytes). Bluetooth and similar protocols allow instructors and students to transmit tutoring modules to each other. An obvious approach to harness this capability would be to combine it with the capabilities of a system such as EDUCA, as they have complementary scope. This approach eliminates the need for significant remote downloading at all, through the use of direct mobile-to-mobile data transfer.

\section{Internet Reliability}

Internet reliability matters the most in a classroom setting, since a short disruption would be a minor hiccup for independent work. However, losing the Internet in a class setting will wreck any lesson plan that relies on it. The systematic study provided few solutions for Internet unreliability. Nedungadi and Raman (2012) employed asynchronous communication for robustness against Internet problems in a mobile context, but this is only useful for web homework or independent study. As a result, 
web-reliant ITS are probably a bad fit for most developing world classrooms. However, web-based ITS could still be effective outside of a school setting if their data usage is handled appropriately. The "well model," Learning Vans (Zualkernan and Karim 2013), and other approaches noted previously should also mitigate these issues.

\section{Electricity}

Only three papers reviewed addressed electricity, in any capacity. Each of these papers noted electricity as a challenge for educational technology, either due to cost issues or inconsistency. Cost issues were noted when using Project LISTEN's Reading Tutor in Zambia, which was the poorest country hosting ITS research (Mills-Tettey et al. 2009). In that study, one school out of two was unable to consistently power their computer lab. The Braille Tutor, an ITS for braille literacy, also considered electricity costs Kalra et al. (2009). This project developed a specialized, low-power "E-Slate" intended to run for 50 hours on a pair of AA batteries.

However, specialized hardware to accommodate lack of electricity is seldom needed. When electricity is irregular, hardware with battery power (e.g., laptops and mobile devices) can mitigate short-term power disruptions with no added cost over desktops. For example, the One Laptop Per Child (OLPC) project initially designed each laptop with hand cranks for power (Patra et al. 2007). Later designs dropped the crank because most users had access to some power source (e.g., community center, generator, car battery). However, Kumar et al. (2012) noted that members of poorer households suffered greater incidents of battery failure for mobile devices, possibly due to heat (summer weather) or bad electrical sources (e.g., spikes). It was also noted that some of the phones may have been damaged due to improper storage (e.g., girls hiding a phone in an oven to prevent their brothers from taking it). While heat can reduce the useable life of batteries, it is unclear how significant this problem will be for educational technology.

\section{Cultural and Language Localization}

Culture and language are combined because the literature seldom addresses culture without addressing language. The most endemic examples of localization occur between or within most-developed countries, such as switching between US and UK English or supporting multicultural regions and institutions. Localization expands beyond language to icons, graphics, and mother media. Localization and supporting users with different native languages have been addressed by a few established ITS architectures. All of these ITS were localized manually. Cognitive Tutor was localized into Spanish and Portuguese by working with local teachers to revise each problem (Ogan et al. 2012). REAP (REAder-specific Practice) was extended from English to Portuguese by researchers who created an equivalent vocabulary list and extended the ITS (Silva et al. 2011). TLCTS (Tactical Language and Culture Training System) worked on the opposite issue: localizing training scenarios to support US soldiers' learning of different cultures (Johnson 2010). 
These accounts all involve skilled local or expert involvement in the project. It is unclear if more efficient alternative practices are possible. Design patterns that separate graphics and text as replaceable assets can ease this process. For example, graphics should not include text because a duplicate graphic would be needed for each language. Web applications and learning management systems standards (e.g., SCORM; scorm.com) support multiple languages by adding markup that designates the language and dialect using International Organization for Standardization (ISO) standards (ISO-639; www.iso.org/iso/home/standards/language_codes. htm). Open standards such as gettext have also been designed for cross-language code development and applications (www.gnu.org/software/gettext/).

However, content extends beyond basic translation and graphics. Local knowledge and cultural understanding are key barriers. Crowd-sourcing services have been used to tag other ITS content, but these techniques have not been explored for ITS localization (Parent and Eskenazi 2010). Services such as Amazon's Mechanical Turk (www.mturk.com/mturk) could provide a low-cost solution to localize content or verify that content has been localized effectively. However, if low cost leads to low quality, crowd sourcing will be ineffective. Research is needed to determine how crowd-sourcing could support high-quality localization, such as localization tasks, processes to monitor reliability (e.g., multiple raters), and boundary conditions (e.g., which content needs expert input).

A final model for localizing an ITS is to localize only the authoring tools, documentation, and certain code functionality (e.g., character sets), rather than any of the content. This approach has been used successfully by the open-source KDE operating system environment (www.kde.org), which is available in over 100 languages. In the ITS community, AutoTutor Lite (Hu et al. 2009) has a Chinese-language prototype that is under development. This version uses authoring tools and documentation translated into Chinese, and Chinese natural language processing functionality is being refined. Compared to content, authoring tools require less localization effort. However, this approach requires local educators or researchers to generate their own tutoring content. This has two major pitfalls. First, tutoring content must be almost completely redeveloped. Since content is not reused, this shifts labor and time costs to local developers. As such, localization effort is shifted from paid experts adapting content to independent authors creating their own ITS content. Second, if local stakeholders struggle to create effective content, it may reflect badly on the tutoring system. Significant training might be required to help local developers author effectively. However, this loss of control is also an advantage. Local developers and authors gain the ability to develop and prototype ITS lessons based on their own goals. Given the freedom to develop their own ITS content, local stakeholders can address topics that developers from most-developed countries did not consider or were uninterested in designing.

\section{ITS by and for Developing Countries}

There are significant differences in the design of tutoring systems intended for developing regions, which depend partly on the stakeholders involved. Three patterns were 
observed among the papers reviewed. In the first pattern, an established ITS from a higher-income country was transferred and localized for one or more less developed countries. These systems tended to be mature, with numerous published papers and evaluations. The second pattern involved a home-grown system developed by researchers in a developing region targeting local or national needs. In the final pattern, a new system designed predominantly in a most-developed country but was intended for a less developed area, as part of a collaboration or ties to the target region. Due to the low number of collaborative and transferred systems combined, these two will be grouped together.

Table 2 breaks down the papers on tutoring systems by the countries involved. As with Table 1, each cell displays the percentage of papers from each group that addressed each barrier. The first row shows the baseline of papers about tutoring systems from high-income countries that address high-income countries. These results are very similar to the "All ITS Papers" results from Table 1, because they have a very high overlap ( $83 \%)$. The next row displays the results for the small group of papers that described an established ITS which had key stakeholders from a high-income country and from a lower-income country. These were either collaborations or adaptations of existing ITS that were transferred to a developing country. Only a handful of papers fell into this category. In the final row, the percentages for home grown ITS from developing countries are displayed. A significant number of papers described home-grown ITS, with China, India, and Mexico represented most strongly.

Across these categories there were some notable differences. Collaborations and transfers of ITS had a higher percentage of papers addressing most barriers. In many ways, this is unsurprising: many of these ITS were primarily designed in high-income countries but deployed in low resource areas. Examples of such ITS were Cognitive Tutor in Latin America, the LISTEN Reading Tutor in Africa, the Single Display Groupware system, and the Braille Tutor (Alcoholado et al. 2012; Kalra et al. 2009; Mills-Tettey et al. 2009; Ogan et al. 2012). Mobile platforms were the major missing piece for these systems: none of the papers reviewed addressed mobile learning or data costs. Overall, even among these systems, the number of papers addressing each barrier was fairly low. This indicates that the level of attention is either still fairly low, or that researchers did not report on their challenges on these topics (e.g., they were not suitable for the publication venue).

Home-grown ITS were quite different. Fewer papers addressed multi-user ITS, infrastructure, culture, or multi-language support. More papers addressed mobile platforms, data costs, and student basic ICT skills. Of these, mobile platforms had the

Table 2 Percentages of ITS Addressing Developing World Barriers, By Economic Status

\begin{tabular}{|c|c|c|c|c|c|c|c|c|c|}
\hline & $\mathrm{N}$ & $\begin{array}{l}\text { Student } \\
\text { ICT }\end{array}$ & $\begin{array}{l}\text { Hardware } \\
\text { Sharing }\end{array}$ & Mobile & $\begin{array}{l}\text { Data } \\
\text { Costs }\end{array}$ & $\begin{array}{l}\text { Electric } \\
\text { Infra. }\end{array}$ & $\begin{array}{l}\text { Internet } \\
\text { Infra. }\end{array}$ & Culture & $\begin{array}{l}\text { Multiple } \\
\text { Languages }\end{array}$ \\
\hline MDC Only & 673 & $1.34 \%$ & $0.59 \%$ & $4.75 \%$ & $0.45 \%$ & $0.00 \%$ & $0.74 \%$ & $4.46 \%$ & $3.71 \%$ \\
\hline $\begin{array}{c}\text { Collaboration } \\
\text { or Transfer }\end{array}$ & 14 & $35.7 \%$ & $28.6 \%$ & $0.00 \%$ & $0.00 \%$ & $14.3 \%$ & $7.14 \%$ & $35.6 \%$ & $28.6 \%$ \\
\hline Home Grown & 128 & $3.13 \%$ & $0.00 \%$ & $11.7 \%$ & $0.78 \%$ & $0.00 \%$ & $0.00 \%$ & $3.91 \%$ & $2.34 \%$ \\
\hline
\end{tabular}


greatest focus by far and touched a number of countries, including Mexico (Cabada et al. 2011), Vietnam (Nguyen and Pham 2011), Jamaica (Henry and Sankaranarayanan 2009), and China (Tam 2012; Wang et al. 2009; Zeng and Lin 2011). This indicates that mobile devices are feasible platforms in many developing regions, but they are underrepresented among ITS from most developed nations building ITS for or with developing nations. Mobile-based ITS research is still a niche in the developing world, but it already plays a greater role (proportionally) than in most-developed countries.

\section{Conclusions and Future Directions}

New opportunities for intelligent tutoring systems are opening up in the developing world, due to changes in ICT availability as well as recent research seeking solutions to developing world barriers. While only a small portion of recent ITS research has addressed these barriers, these papers have outlined possible solutions to many of these issues. This study summarized these barriers and existing solutions, with the hope that later projects can leverage these solutions.

Across the papers reviewed, the prevalence of both barriers and solutions is likely understated. The reasons behind this are multi-faceted. First, some of these barriers are prone to selection bias: researchers implement their tutoring systems in classrooms that meet the systems' basic requirements (e.g., sufficient computers, stable electricity, Internet access). As a result, the true prevalence of barriers will be consistently understated. Second, many of these topics are often considered throwaway "implementation details." This is somewhat ironic: implementation factors are seldom studied systematically because they require large-scale, multi-site studies. Since most studies only have one site, their details are often under-reported, even though such data is hard to collect. In aggregate, the implementation considerations of a large number of varied implementations can offer significant insight, but such data often has no clear home.

This is due to a larger pattern of specialization: as the overall output of research articles has increased, most journals and conferences have grown into separate silos segmented by subdiscipline (Silverman 2010) and nationality (Tight 2014). Venues outside of education (e.g., computer science, psychology), and even some inside education, may feel that their readers will not be interested in a discussion of implementation challenges and solutions. Unfortunately, researchers who publish primarily in such venues may not find time to publish such details elsewhere, discarding casestudy data on barriers to adoption. Journal editors may be able to mitigate such losses. For each study in a real educational context (e.g., field study), at least one paragraph should explicitly outline barriers that impacted the system design or implementation. If this minimal standard was held across venues, a clearer picture of stumbling blocks and usability issues would emerge.

Despite the limitations of the available data, meaningful patterns were observed. While these barriers were examined from the standpoint of ITS, they are also relevant to other educational technologies and contexts. The greater prevalence of mobile-based ITS in developing contexts may be evidence of a partial leap-frogging 
of traditional computer-based ITS or a move toward ubiquitous ITS systems that span multiple platforms. However, this possibility has two significant caveats. First, home-grown ITS were more likely to target tertiary education, such as universities. Universities are more likely to have effective technology infrastructure and support, which may make mobile learning more feasible. Second, work on mobile learning was dominated by large emerging economies (e.g., Mexico, India). These countries have large enough internal disparities that mobile ITS might only be feasible in certain regions or communities. With that said, a mobile ITS only feasible for one-third of India would still serve a population the size of the United States.

When considering mobile ITS, a significant issue exists for targeting new research projects: How advanced will mobile phones be in a region by the time a certain educational technology is ready? At present, mobile broadband still has a fairly low penetration in developing countries $(21 \%)$. Of such subscriptions, not all users are accessing using smartphones. Based on logistic models commonly used to model diffusion of innovation (Rogers 1995), Africa does not project to have $50 \%$ smartphone penetration until 2020, but other continents are projected to exceed that level by the end of 2016 (Dedui 2014). However, researchers should probably avoid using aggregate numbers, even at the national level, for considering mobile ITS. There are wide differences in smartphone adoption across countries, as well as strong urban-rural and age-based divides, where younger city-dwellers tend to adopt earlier (International Telecommunication Union 2014). In a given region, a three-year research project could probably see a shift from adoption levels of $20 \%$ to well over $50 \%$ in some cities. For new projects in most regions, smartphones are likely a better platform than feature phones. Nokia's feature phones have previously been a major part of the market, but these are being displaced by low-cost smartphones. Since Nokia moved from its proprietary Symbian OS to Windows Phone OS, three smartphone operating systems cover nearly the entire market (Apple iOS, Google Android, and Windows Phone), and iOS phones have lower adoption in developing regions. However, if targeting feature phones, it should be noted that most feature phones now support Java ME for third-party applications, providing a (somewhat) common platform.

A second conclusion from this study was that educational context is very important when considering barriers to adoption. Barriers to classroom use are quite different from home use, which calls for different models of technology for these settings. For classroom use, shared laptops running installed software show promise. For independent use, mobile applications downloaded at community centers or mobile-to-mobile over Bluetooth might be more accessible. In either context, language and cultural localization are important to adoption. Future research may address such questions as: How do multiple-input devices impact user models? How might existing ITS and other technologies be adapted for the mobile interfaces and hardware capabilities? Can parts of localization be automated? Because developing nations have pressing educational needs and existing studies present a culturally-biased sample due to under-representation of these areas (Blanchard 2012), increased focus on ITS and other learning technologies for the developing world is warranted.

Finally, while the developing world has unique barriers to adoption of ITS, the most challenging barriers may be the ones that are shared by all countries. Teacher beliefs on ICT, the pedagogical match of the ITS to teachers, limited training time, 
curriculum restrictions, and many other barriers have impacted educational technology in most-developed and developing countries (Bingimlas 2009; Gulati 2008). The level of attention by ITS to these barriers must be evaluated, to identify challenges and potential solutions. For this reason, this analysis is continuing as a mapping study to consider recent developments in ITS, with a special focus on barriers to adoption. Current work is exploring barriers to adoption identified predominantly in the most developed countries, which also are likely to impact ITS in the developing world. Additionally, a website is under development to easily browse and download the metadata accumulated through this study. This site is intended to facilitate locating ITS papers that address particular barriers to ITS adoption, as well as consider trends in ITS publishing. Hopefully, this site should help scholars locate relevant research and potential collaborators in the worldwide ITS community.

\section{Appendix: Papers Meeting Inclusion Criteria by Country}

Table 3 Counts of Papers Meeting Inclusion Criteria, By Country (Sorted by Total)

\begin{tabular}{|c|c|c|c|}
\hline Country & Total Papers & ITS Papers & Adaptive Systems Papers \\
\hline USA & 393 & 373 & 20 \\
\hline China & 67 & 44 & 23 \\
\hline Spain & 55 & 23 & 32 \\
\hline UK & 54 & 44 & 10 \\
\hline Canada & 54 & 50 & 4 \\
\hline Taiwan & 50 & 27 & 23 \\
\hline Germany & 30 & 24 & 6 \\
\hline Mexico & 29 & 23 & 6 \\
\hline Greece & 29 & 19 & 10 \\
\hline India & 27 & 20 & 7 \\
\hline Brazil & 22 & 13 & 9 \\
\hline France & 20 & 14 & 6 \\
\hline Italy & 19 & 7 & 12 \\
\hline New Zealand & 18 & 18 & 0 \\
\hline Netherlands & 18 & 12 & 6 \\
\hline Portugal & 15 & 13 & 2 \\
\hline Australia & 15 & 12 & 3 \\
\hline Japan & 13 & 6 & 7 \\
\hline Serbia & 10 & 4 & 6 \\
\hline Malaysia & 10 & 10 & 0 \\
\hline Korea & 9 & 7 & 2 \\
\hline Austria & 9 & 3 & 6 \\
\hline Philippines & 8 & 8 & 0 \\
\hline Turkey & 6 & 3 & 3 \\
\hline Tunisia & 6 & 3 & 3 \\
\hline
\end{tabular}


Table 3 (continued)

\begin{tabular}{|c|c|c|c|}
\hline Country & Total Papers & ITS Papers & Adaptive Systems Papers \\
\hline Ireland & 6 & 4 & 2 \\
\hline Thailand & 5 & 4 & 1 \\
\hline Switzerland & 5 & 3 & 2 \\
\hline Singapore & 5 & 4 & 1 \\
\hline Pakistan & 5 & 3 & 2 \\
\hline Croatia & 5 & 3 & 2 \\
\hline Chile & 5 & 5 & 0 \\
\hline Latvia & 4 & 2 & 2 \\
\hline Iran & 4 & 0 & 4 \\
\hline Czech Republic & 4 & 4 & 0 \\
\hline Colombia & 4 & 2 & 2 \\
\hline Slovakia & 3 & 3 & 0 \\
\hline Norway & 3 & 0 & 3 \\
\hline Egypt & 3 & 1 & 2 \\
\hline Saudi Arabia & 2 & 0 & 2 \\
\hline Denmark & 3 & 3 & 0 \\
\hline Costa Rica & 3 & 2 & 1 \\
\hline Zambia & 2 & 2 & 0 \\
\hline Vietnam & 2 & 1 & 1 \\
\hline \multicolumn{4}{|l|}{ and Tobago } \\
\hline Romania & 2 & 0 & 2 \\
\hline Poland & 2 & 1 & 1 \\
\hline Morocco & 2 & 0 & 2 \\
\hline Finland & 2 & 2 & 0 \\
\hline Belgium & 2 & 1 & 1 \\
\hline Algeria & 2 & 0 & 2 \\
\hline United Arab & 1 & 0 & 1 \\
\hline \multicolumn{4}{|l|}{ Emirates } \\
\hline Sweden & 1 & 1 & 0 \\
\hline Sri Lanka & 1 & 1 & 0 \\
\hline Slovenia & 1 & 1 & 0 \\
\hline Qatar & 1 & 1 & 0 \\
\hline Luxembourg & 1 & 0 & 1 \\
\hline Libya & 1 & 0 & 1 \\
\hline Jamaica & 1 & 1 & 0 \\
\hline Guatemala & 1 & 1 & 0 \\
\hline Ghana & 1 & 1 & 0 \\
\hline
\end{tabular}




\section{References}

Alcoholado, C., Nussbaum, M., Tagle, A., Gomez, F., Denardin, F., Susaeta, H., et al. (2012). One mouse per child: Interpersonal computer for individual arithmetic practice. Journal of Computer Assisted Learning, 28(4), 295-309.

Balanskat, A., Blamire, R., Kefala, S. (2006). The ICT impact report: A review of studies of ICT impact on schools in Europe: Brussels European Schoolnet.

Banerjee, A., Cole, S., Duflo, E., Linden, L. (2007). Remedying education: Evidence from two randomized experiments in India. The Quarterly Journal of Economics, 122(3), 1235-64.

Bingimlas, K. (2009). Barriers to the successful integration of ICT in teaching and learning environments: A review of the literature. Eurasia Journal of Mathematics, Science and Technology Education, 5(3), $235-245$.

Blanchard, E.G. (2012). On the WEIRD nature of ITS/AIED conferences: A 10 year longitudinal study analyzing potential cultural biases. Intelligent Tutoring Systems (ITS) 2012, (pp. 280-285). Berlin: Springer.

Bransford, J., Brown, A., Cocking, R. (2000). How people learn. Washington, DC: National Academy Press.

Bronfenbrenner, U. (1992). Ecological systems theory: Jessica Kingsley Publishers.

Brunskill, E., Garg, S., Tseng, C., Findlater, L. (2010). Evaluating an adaptive multi-user educational tool for low-resource environments. Information and Communication Technologies for Development (ICTD) 2010. London: IEEE Press.

Cabada, R.Z., Barrón Estrada, M.L., Reyes García, C.A. (2011). EDUCA: A web 2.0 authoring tool for developing adaptive and intelligent tutoring systems using a Kohonen network. Expert Systems with Applications, 38(8), 9522-9529.

Cabada, R.Z., Estrada, M.L.B., Parra, L.E., Garcia, C.A.R. (2011). Interpreter for the deployment of intelligent tutoring systems in mobile devices. International Conference on Advanced Learning Technologies (ICALT) 2011, (pp. 339-340). Washington, DC: IEEE Press.

Casas, I., Goodman, P.S., Pelaez, E. (2011). On the design and use of a cognitive tutoring system in the math classroom. Technology for Education (T4E) 2011, (pp. 9-17). Piscataway, NJ: IEEE Press.

Cassim, K.M., \& Eyono Obono, S.D. (2011). On the factors affecting the adoption of ICT for the teaching of word problems. In Ao, S.I., Douglas, C., Grundfest, W.S., Burgstone, J. (Eds.) World Congress on Engineering and Computer Science 2011, Vol. 1, (pp. 269-276). San Francisco, CA: Newswood Limited.

Chu, H.C., Hwang, G.J., Tsai, C.-C., Tseng, J.C.R. (2010). A two-tier test approach to developing locationaware mobile learning systems for natural science courses. Computers and Education, 55(4), 16181627.

Dedui, H. (2014). When will smartphones saturate. Retrieved: June 18, 2014. www.asymco.com/2014/01/ 07/when-will-smartphones-saturate/.

Eco, U. (1979). A theory of semiotics: Indiana University Press.

Gitau, S., Marsden, G., Donner, J. (2010). After Access: Challenges Facing Mobile-Only Internet Users in the Developing World. In SIGCHI, (pp. 2603-06). New York, NY: ACM Press.

Goktas, Y., Yildirim, S., Yildirim, Z. (2009). Main Barriers and Possible Enablers of ICTs Integration into Pre-Service Teacher Education Programs. Educational Technology and Society Society, 12(1), 193-204.

Graesser, A., Chipman, P., Haynes, B., Olney, A. (2005). AutoTutor: An Intelligent Tutoring System With Mixed-Initiative Dialogue. IEEE Transactions on Education, 48(4), 612-618.

Gulati, S. (2008). Technology-enhanced learning in developing nations: A review. International Review of Research in Open and Distance Learning, 9(1), 1-16.

Henry, L., \& Sankaranarayanan, S. (2009). Application of intelligent agents for mobile tutoring. In Proceedings of the 2nd International Conference on Interaction Sciences: Information Technology, Culture and Human, (pp. 963-969). New York, NY: ACM Press.

Hu, X., Cai, Z., Han, L., Craig, S.D., Wang, T., Graesser, A.C. (2009). AutoTutor Lite. In: AIED 2009. Amsterdam, The Netherlands: IOS Press.

International Telecommunication Union (2012). Measuring the Information Society. Geneva, Switzerland. International Telecommunication Union (2014). ICT Facts and Figures. Geneva, Switzerland.

Johnson, W.L. (2010). Serious Use of a Serious Game for Language Learning. International Journal of Artificial Intelligence in Education, 20(2), 175-195. 
Kalra, N., Lauwers, T., Dewey, D., Stepleton, T., Dias, M.B. (2009). Design of a braille writing tutor to combat illiteracy. Information Systems Frontiers, 11(2), 117-128.

Koedinger, K.R., \& Corbett, A.T. (2006). Cognitive tutors : technology bringing learning science to the classroom. In Sawyer, R.K. (Ed.) The Cambridge handbook of the learning sciences, (pp. 61-77). New York, NY: Cambridge University Press.

Kumar, A., Reddy, P., Tewari, A., Agrawal, R., Kam, M. (2012). Improving literacy in developing countries using speech recognition-supported games on mobile devices. SIGCHI, (pp. 1149-1158): ACM Press.

Lowther, D.L., Inan, F.A., Strahl, J.D., Ross, S.M. (2008). Does technology integration "Work" when key barriers are removed?. Educational Media International, 45(3), 195-213.

McKnight, L., \& Read, J.C. (2009). Designing the 'record' button. Proceedings of the International Conference on Interaction Design and Children (IDC) 2009, (pp. 258-261). New York, NY: ACM Press.

Mills-Tettey, G.A., Mostow, J., Dias, M.B., Sweet, T.M., Belousov, S.M., Dias, M.F., et al. (2009). Improving child literacy in africa: Experiments with an automated reading tutor. Information and Communication Technologies for Development (ICTD) 2009, (pp. 129-138). Piscataway, NJ: IEEE Press.

Mitra, S., \& Dangwal, R. (2010). Limits to self-organising systems of learning- The Kalikuppam Experiment. British Journal of Educational Technology, 41(5), 672-688.

Munoz-Organero, M., Munoz-Merino, P.J., Kloos, C.D. (2012). Sending learning pills to mobile devices in class to enhance student performance and motivation in network services configuration courses. IEEE Transactions on Education, 55(1), 83-87.

Nedungadi, P., \& Raman, R. (2012). A New Approach to Personalization: Integrating E-Learning and M-Learning. Educational Technology Research and Development, 60(4), 659-678.

Nguyen, V.A., \& Pham, V.C. (2011). Bayesian network to manage learner model in context-aware adaptive system in mobile learning. Proceedings of the 6th international conference on E-learning and games, edutainment technologies, (pp. 63-70). Berlin, Heidelberg: Springer.

Ogan, A., Walker, E., Baker, R.S., Rebolledo Mendez, G., Jimenez Castro, M., Laurentino, T. (2012). Collaboration in cognitive tutor use in Latin America: Field study and design recommendations. SIGCHI 2012, (pp. 1381-1390). New York, NY: ACM Press.

Parent, G., \& Eskenazi, M. (2010). Clustering dictionary definitions using Amazon Mechanical Turk. 2010 NAACL HLT Workshop on Creating Speech and Language Data with Amazon's Mechanical Turk. Stroudsburg, PA, USA: Association for Computational Linguistics.

Patra, R., Pal, J., Nedevschi, S., Plauche, M., Pawar, U. (2007). Usage models of classroom computing in developing regions. Information and Communication Technologies for Development (ICTD) 2007 , (pp. 158-167). Piscataway, NJ: IEEE Press.

Petersen, K., Feldt, R., Mujtaba, S., Mattsson, M. (2008). Systematic mapping studies in software engineering. EASE 2008, (pp. 1-8): IET Publications.

Population Reference Bureau (2012). 2012 World Population Data Sheet. Washington, DC.

Puntambekar, D.M., Gondal, S., Agrawal, M. (2009). Province of multiuser m-learning environment using artificial intelligence: bluetooth techniques. In Asian Himalayas International Conference on Internet (AH-ICI) 2009, (pp. 1-5). Piscataway, NJ: IEEE Press.

Riasati, M.J., Allahyar, N., Tan, K.E. (2012). Technology in language education: Benefits and barriers. Journal of Education and Practice, 3(5), 25-30.

Rogers, E.M. (1995). Diffusion of innovations: Free Press.

Savvopoulos, A., \& Virvou, M. (2010). Tutoring the elderly on the use of recommending systems. CampusWide Information Systems, 27(3), 162-172.

Silva, A., Mamede, N., Ferreira, A., Baptista, J., Fernandes, J. (2011). Towards a serious game for portuguese learning. Serious Games Development and Applications (SGDA) 2011, (pp. 83-94). Heidelberg, Germany: Springer.

Silverman, B.G. (2010). Systems social science: A design inquiry approach for stabilization and reconstruction of social systems. Intelligent Decision Technologies, 4(1), 51-74.

Tam, V. (2012). An intelligent e-learning software for learning to write correct chinese characters on mobile devices. Interactive Technology and Smart Education, 9(4), 191-203.

Tight, M. (2014). Working in separate silos? what citation patterns reveal about higher education research internationally. Higher Education. 
VanLehn, K. (2006). The behavior of tutoring systems. International Journal of Artificial Intelligence in Education, 16(3), 227-265.

VanLehn, K. (2011). The relative effectiveness of human tutoring, intelligent tutoring systems, and other tutoring systems. Educational Psychologist, 46(4), 197-221.

Virvou, M., Alepis, E., Troussas, C. (2012). A mobile expert system for tutoring multiple languages using machine learning. In International Conference on e-learning and e-technologies in Education (ICEEE) 2012, (pp. 128-133). IPM.

Vygotsky, L.S. (1980). Mind in society. Cambridge, MA: Harvard University Press.

Wang, Z., Qiao, X., Xie, Y. (2009). An emotional intelligent e-learning system based on mobile agent technology. International conference on computer engineering and technology (ICCET) 2009 Vol. 1, (pp. 51-54). Piscataway, NJ: IEEE Press.

World Bank (2013a). Country and lending groups. Retrieved: Dec. 28, 2013. data.worldbank.org/about/ country-classifications/country-and-lending-groups.

World Bank (2013b). World databank . Retrieved: December 8, 2013. databank.worldbank.org/data/.

Zeng, L., \& Lin, L. (2011). An interactive vocabulary learning system based on word frequency lists and ebbinghaus' curve of forgetting. Digital Media and Digital Content Management (DMDCM) 2011 Workshop, (pp. 313-317). Piscataway, NJ: IEEE Press.

Zualkernan, I.A., \& Karim, A. (2013). Using a traveling van to deliver blended learning in a developing country. International Conference on Advanced Learning Technologies (ICALT) 2013, (pp. 429-431). Piscataway, NJ: IEEE Press. 